\title{
Economic and Humanistic Burden of Uterine Cancer in the United States
}

Nwankwo C1; Shah R2; Kwon Y2; Corman $\mathrm{S}^{2}$

${ }^{1}$ Merck \& Co., Inc., Kenilworth, NJ, USA; 2Pharmerit International LP, Bethesda, MD, USA

\section{BACKGROUND}

- Uterine cancer is the most common cancer of the female reproductive system. New diagnoses of uterine cancer number 25.7 per 100,000 per year, of which the majority are adenocarcinomas of the endometrium

- Little is known about the economic and humanistic burden of endometrial cancer in the US

There have been no published studies on healthcare costs, and the quality of life of uterine cancer patients is described in only select populations such as long-term survivors and those undergoing nerve-sparing radical hysterectomy ${ }^{3}$

\section{OBJECTIVE}

The objective of this study was to describe economic and humanistic burden of uterine cancer patients in the United States, using a nationally representative dataset

\section{METHODS}

This was a retrospective, cross-sectional analysis of the Medical Expenditure Panel Survey (MEPS) data from 2006-2015

Uterine cancer patients were identified using ICD-9 CM code 182 or Clinical Classification Software code 25. Patients with a diagnosis of another cancer in addition to uterine cancer were excluded. The control group consisted of women without a diagnosis of cancer

Study outcomes included the following: Healthcare resource use (number of prescriptions, institutional inpatient and outpatient, ER, and physician office visits) Healthcare costs

Activities of daily living (physical, cognitive, social, and activity limitations)

Quality of life measures (SF-12v2 physical component score [PCS], mental component score [MCS], EQ-5D health utility, and PHQ-2 depression severity)

Unadjusted bivariate analyses were conducted using t-tests for continuous variables and chisquare tests for categorical variables

Multivariate generalized linear models (GLM) that controlled for key sociodemographic and clinical covariates were conducted for adjusted comparisons of study outcomes between uterine cancer patients and non-cancer controls

All analyses used appropriate procedures to account for the complex survey design of the MEPS

\section{RESULTS}

Patient Characteristics

- The final cohort consisted of 269,907 uterine cancer patients (unweighted frequency: 253) and $146,061,609$ non-cancer controls (unweighted frequency: 171936) (Table 1)

Uterine cancer patients were significantly older (mean age: 60.8 vs 37.0 years), had a highe BMI (mean BMI: 31.3 vs $26.5 \mathrm{~kg} / \mathrm{m}^{2}$ ), and greate comorbidity burden (mean Charlson comorbidity index: 1.5 vs 0.5 ) compared to controls

A greater portion of uterine cancer patients had Aair/poor perceived general and mental health status than that of controls
Table 1. Patient Demographic and Clinical Characteristics

\begin{tabular}{|c|c|c|}
\hline \multirow[b]{2}{*}{ Statistic or Category } & Uterine Cancer & Non-Cancer \\
\hline & $(\mathrm{N}=269,907)$ & $(\mathrm{N}=146,061,609)$ \\
\hline Mean agea & $60.82(1.59)$ & $36.98(0.18)$ \\
\hline \multicolumn{3}{|l|}{ Race/ethnicitya } \\
\hline White & $234,713(87.0 \%)$ & $113,614,171$ (77.8\% \\
\hline Black & $14,970(5.6 \%)$ & $19,683,216(13.5 \%$ \\
\hline $\begin{array}{l}\text { American Indian/ } \\
\text { Alaskan Native }\end{array}$ & $1,698(0.6 \%)$ & $1,139,810(0.8 \%)$ \\
\hline $\begin{array}{l}\text { Asian/Native Hawaiian/ } \\
\text { Pacific Islanders }\end{array}$ & $2,895(1.1 \%)$ & $8,227,062(5.6 \%)$ \\
\hline Multiple race & $15,632(5.8 \%)$ & $3,397,350(2.3 \%)$ \\
\hline \multicolumn{3}{|l|}{ Educationa } \\
\hline 1st-8th grade & $12,352(4.6 \%)$ & $26,136,352(17.9 \%$ \\
\hline $\begin{array}{l}\text { 9th-12th grade } \\
\text { (no HS diploma/GED) }\end{array}$ & $64,721(24.0 \%)$ & $31,000,110(21.2 \%$ \\
\hline GED/HS grad & $76,399(28.3 \%)$ & $24,083,436(16.5 \%$ \\
\hline Beyond HS/associate degree/college & $58,967(21.8 \%)$ & $21,508,455(14.7 \%$ \\
\hline BA/4-year college degree & $53,444(19.8 \%)$ & $19,430,309(13.3 \%$ \\
\hline Masters/doctorate/professional & $3,144(1.2 \%)$ & $10,716,955(7.3 \%)$ \\
\hline \multicolumn{3}{|l|}{ Census regiona } \\
\hline Northeast & $74,164(27.5 \%)$ & $25,987,919(17.8 \%$ \\
\hline Midwest & $55,253(20.5 \%)$ & $31,115,668(21.3 \%$ \\
\hline South & $74,292(27.5 \%)$ & $54,141,696(37.1 \%)$ \\
\hline West & $57,818(21.4 \%)$ & $33,792,238(23.1 \%$ \\
\hline \multicolumn{3}{|l|}{ Insurance statusa } \\
\hline Private & $176,643(65.4 \%)$ & $94,904,453(65.0 \%$ \\
\hline Public only & $80,726(29.9 \%)$ & $35,239,927(24.1 \%$ \\
\hline Uninsured & $12,538(4.7 \%)$ & $15,917,229(10.9 \%$ \\
\hline Mean BMla & $31.25(1.06)$ & $26.45(0.05)$ \\
\hline Mean CCla & $1.46(0.19)$ & $0.46(0.01)$ \\
\hline \multicolumn{3}{|l|}{ General healtha } \\
\hline 1 Excellent & $22,509(8.3 \%)$ & $46,186,208(31.6 \%$ \\
\hline 2 Very good & $68,918(25.5 \%)$ & $47,510,019(32.5 \%$ \\
\hline 3 Good & $96,412(35.7 \%)$ & $36,080,314(24.7 \%$ \\
\hline 4 Fair & $51,357(19.0 \%)$ & $11,434,552(7.8 \%)$ \\
\hline 5 Poor & $14,549(5.4 \%)$ & $3,529,071(2.4 \%)$ \\
\hline \multicolumn{3}{|l|}{ Mental healtha } \\
\hline 1 Excellent & $62,759(23.3 \%)$ & $59,790,006(40.9 \%$ \\
\hline 2 Very good & $71,959(26.7 \%)$ & $42,830,335(29.3 \%$ \\
\hline 3 Good & $81,197(30.1 \%)$ & $32,774,664(22.4 \%$ \\
\hline 4 Fair & $30,635(11.4 \%)$ & $7,467,825(5.1 \%)$ \\
\hline 5 Poor & $7,194(2.7 \%)$ & $1,859,789(1.3 \%)$ \\
\hline
\end{tabular}

GED, general equivalency dip

Statistically significant difference asses

Results are

Economic Burden

Unadjusted bivariate analyses suggested that uterine cancer was associated with significantly higher HCRU and costs (Table 2)

Table 2. Unadjusted HCRU and Costs

\begin{tabular}{|l|c|c|}
\hline \multirow{2}{*}{\multicolumn{1}{|c|}{ Variable Description }} & Uterine Cancer & Non-Cancer \\
\cline { 2 - 3 } & $\mathbf{( N = 2 6 9 , 9 0 7 )}$ & $\mathbf{( N = 1 4 6 , 0 6 1 , 6 0 9 )}$ \\
\hline Number of prescriptions & $30.41(2.77)$ & $10.99(0.14)$ \\
\hline Number of hospitalizations & $0.50(0.06)$ & $0.10(0.00)$ \\
\hline Number of ER visitsa & $0.37(0.06)$ & $0.20(0.00)$ \\
\hline Number of office-based visitsa & $12.67(1.26)$ & $5.56(0.06)$ \\
\hline Number of outpatient visitsa & $1.83(0.27)$ & $0.40(0.01)$ \\
\hline Total healthcare costsa & $\$ 15,337(\$ 1,704)$ & $\$ 3,829(\$ 55)$ \\
\hline Drug costa & $\$ 3,275(\$ 391)$ & $\$ 975(\$ 20)$ \\
\hline Hospitalization costa & $\$ 7,244(\$ 1,340)$ & $\$ 1,225(\$ 34)$ \\
\hline ER cost & $\$ 312(\$ 80)$ & $\$ 188(\$ 4)$ \\
\hline Office-based costa & $\$ 2,855(\$ 291)$ & $\$ 1,070(\$ 13)$ \\
\hline Outpatient costa & $\$ 1,651(\$ 367)$ & $\$ 371(\$ 17)$ \\
\hline
\end{tabular}

Statistically significant difference assessed at $P$-value $=0.05$.

Results from the multivariate GLMs (Figures 1A, 1B) suggested that as compared to non-cancer controls, uterine cancer patients had significantly higher:

Number of inpatient visits $(0.3$ vs 0.1$)$ and costs $(\$ 6,117$ vs $\$ 1,446)$ Outpatient physician visits $(0.9$ vs 0.5$)$ and costs $(\$ 1,229$ vs $\$ 502)$ Institutional outpatient costs ( $\$ 1,965$ vs $\$ 1,322)$

Total all-cause healthcare costs $(\$ 11,490$ vs $\$ 4,909$ )
Figure 1A. Adjusted Healthcare Resource Use from GLM

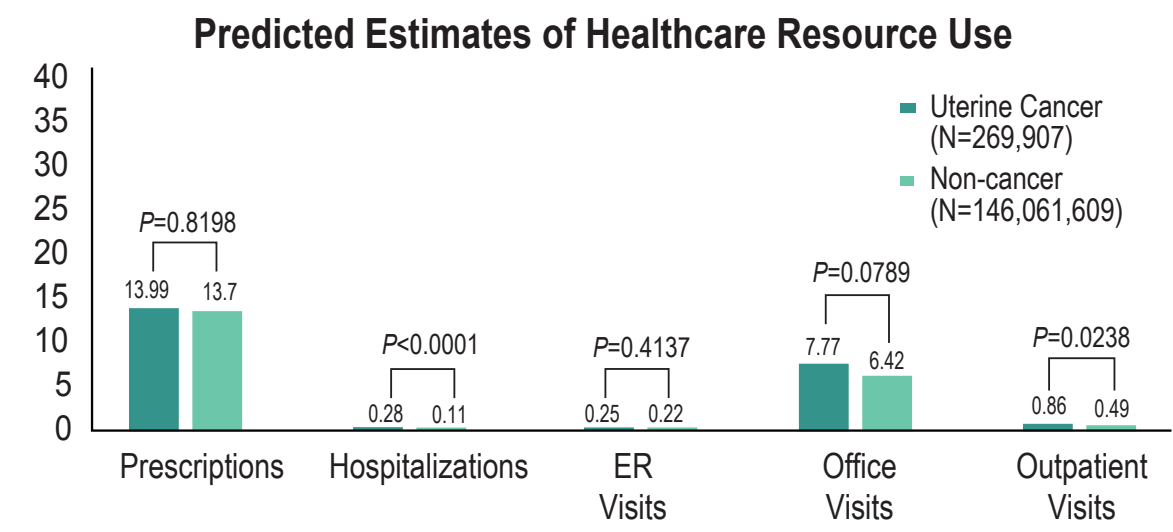

Figure 1B. Adjusted Healthcare Costs from GLM

Predicted Estimates of Healthcare Costs

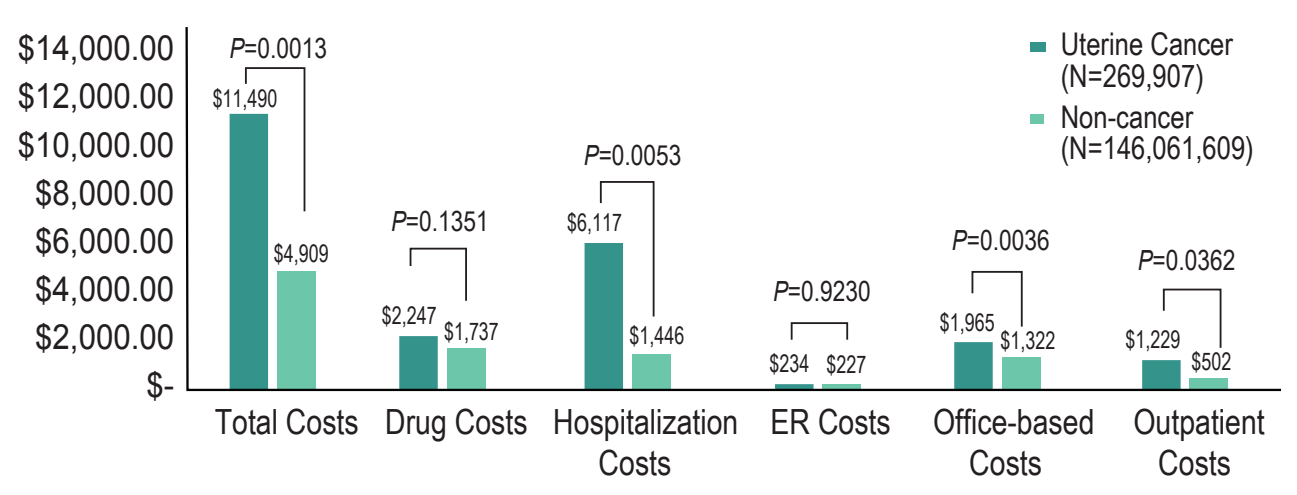

Humanistic Burden

In the unadjusted analysis, physical, activity, social, and cognitive limitations were significantly higher while EQ-5D utility scores were significantly lower for uterine cancer patients compared to non-cancer controls (Table 3 )

In the adjusted analyses, uterine cancer patients did not significantly differ

from the non-cancer controls in terms of functional and general health status

(Figure 2, Figure 3)

Uterine cancer was significantly associated with lower EQ-5D utility (Beta coefficient $=-0.273 ; P=0.013)$ compared to non-cancer controls $(P<0.05)$

Table 3. Unadjusted Analysis of PRO Measures in Uterine Cancer Population

\begin{tabular}{|l|c|c|}
\hline \multicolumn{1}{|c|}{ Variable Description } & Uterine Cancer & Non-Cancer \\
\cline { 2 - 3 } & $(\mathrm{N}=269,907)$ & $(\mathrm{N}=146,061,609)$ \\
\hline Physical limitations, $\mathrm{N}(\%)^{a}$ & $84,171(31.2 \%)$ & $14,877,178(10.2 \%)$ \\
\hline Activity limitations, $\mathrm{N}(\%)^{a}$ & $60,357(22.4 \%)$ & $10,976,127(7.5 \%)$ \\
\hline Social limitations, $\mathrm{N}(\%)^{\mathrm{a}}$ & $36,217(13.4 \%)$ & $6,226,559(4.3 \%)$ \\
\hline Cognitive limitations, $\mathrm{N}(\%)^{\mathrm{a}}$ & $21,173(7.8 \%)$ & $5,619,573(3.9 \%)$ \\
\hline SF-12 PCS, Mean (SD) & $41.50(1.08)$ & $49.20(0.08)$ \\
\hline SF-12 MCS, Mean (SD) & $50.32(0.85)$ & $50.40(0.06)$ \\
\hline PHQ-2 score, Mean (SD) & $0.86(0.12)$ & $0.74(0.01)$ \\
\hline SF-6D utility score, Mean (SD) & $0.56(0.01)$ & $0.56(0.00)$ \\
\hline EQ5D utility score, Mean (SD) & $0.79(0.01)$ & $0.86(0.00)$ \\
\hline
\end{tabular}

Figure 2. Adjusted Functional Figure 3. Adjusted SF-12, PHQ-2, Limitation and Health Status and Health Utility Scores

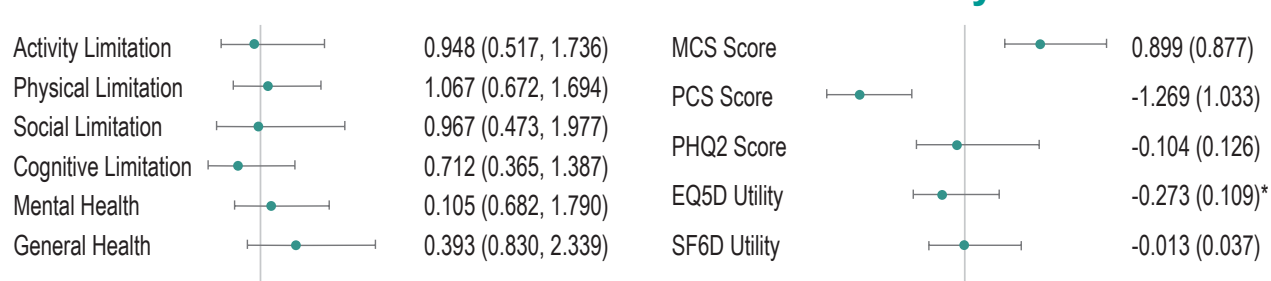

ed at $P$-value $=0.05$

\section{STUDY LIMITATIONS}

The study employed a cross-sectional design, which precludes us from drawing causal inferences about the relationship between uterine cancer and the study outcomes

Other variables such as cancer staging information or time since diagnosis were not available in the data

The detected cancer patients only represent the "treated prevalence" and therefore the cancer patients might have been under- or over-estimated due to potential misclassification issues related to using 3-digit ICD and CCC codes

\section{CONCLUSIONS}

Uterine cancer is associated with significant healthcare resource use and cost burden, and health utility impairment

These results are generalizable to the population of non-institutionalized US adults and should be considered by payers in making resource allocation decisions and by clinicians in drafting uterine cancer screening treatment guidelines

References

National Cancer Institute Surveillance, Epidemiology, and End Results Program (SEER).

Dobrzycka B, et al. Ann Agric Environ Med. 2017:24(3):513-516.

Kruppa J, et al. Eur J Obstet Gynecol Reprod Biol. 2016:201:131-134.

Disclosures

Disclosures 